\title{
Influence of pantograph fixing position on aerodynamic characteristics of high-speed trains
}

\author{
Liang Zhang ${ }^{1} \cdot$ Jiye Zhang ${ }^{1} \cdot$ Tian $\mathrm{Li}^{1} \cdot$ Weihua Zhang ${ }^{1}$
}

Received: 28 September 2016/Revised: 19 January 2017/ Accepted: 19 January 2017/Published online: 28 February 2017

(C) The Author(s) 2017. This article is published with open access at Springerlink.com

\begin{abstract}
To study the influence of the pantograph fixing position on aerodynamic characteristics of high-speed trains, the aerodynamic models of high-speed trains with eight cars were established based on the theory of computational fluid dynamics, and eight cases with pantographs fixed on different positions and in different operational orientations were considered. The pantographs were fixed on the front or the rear end of the first middle car or fixed on the front or the rear end of the last middle car. The external flow fields of the high-speed trains were numerically simulated using the software STAR-CCM+. The results show that the pantograph fixing position has little effect on the aerodynamic drag force of the head car and has a large effect on the aerodynamic drag force of the tail car. The influences of the pantograph fixing position on the aerodynamic lift forces of the head car, tail car and pantographs are obvious. Among the eight cases, considering the total aerodynamic drag force of the train and the aerodynamic lift force of the lifted pantograph, when the pantographs are fixed on the rear end of the last middle car and the lifted pantograph is in the knuckle-upstream orientation, the aerodynamic performance of the high-speed train is the best.
\end{abstract}

Keywords High-speed train · Pantograph · Fixing position - Aerodynamic characteristics . Computational fluid dynamics

Liang Zhang

swjtu.zl@163.com

1 State Key Laboratory of Traction Power, Southwest Jiaotong University, Chengdu 610031, China

\section{Introduction}

With the increase in the train speed, the interaction between the train and the air becomes more severe, and it leads to a series of aerodynamic problems, such as aerodynamic drag force, lift force, aerodynamic noise [1]. The aerodynamic drag force is proportional to the square of the train speed. When the train speed reaches $200-300 \mathrm{~km} / \mathrm{h}$, the aerodynamic drag accounts for $70 \%$ to $85 \%$ of the total drag of the train $[1,2]$. The aerodynamic drag of pantographs accounts for $8 \%$ to $14 \%$ of the total aerodynamic drag, and the aerodynamic lift of pantographs is proportional to the square of the train speed [3]. Thus, the increase in the train speed would lead to a stronger interaction of pantograph-catenary $[4,5]$.

A great deal of research has been carried out to investigate the aerodynamic characteristics of pantographs of high-speed trains. Zhang et al. [6] studied the influence of the fairing and windshield on the aerodynamic drag of pantographs through wind tunnel tests. Guo et al. [7] studied the unsteady aerodynamic characteristics of pantographs of high-speed trains with and without crosswind conditions using the detached eddy simulation method. Li et al. [8] analyzed the aerodynamic forces of pantographs with knuckle-downstream and knuckle-upstream orientation through a numerical simulation based on the threedimensional (3D) steady Reynolds Average Navier-Stokes (RANS) method, and the simulation results were basically consistent with the experimental results. Fu et al. [9] studied the aerodynamic forces of pantographs and the vibration characteristics induced by winds through wind tunnel tests. Pombo et al. [10] analyzed the influence of the aerodynamic forces on the pantograph-catenary system for high-speed trains under crosswinds using numerical simulations and experiments. Lee et al. [11] performed wind 
tunnel tests of pantographs with different arms and optimized the panhead shape. Du et al. [12] numerically calculated the flow field around a pantograph and analyzed the aeroacoustic characteristics of the pantograph.

However, the research about the aerodynamic characteristics of pantographs in the above literature mainly focused on the pantograph itself and neglected the influence of the train body on the aerodynamic characteristics of pantographs. As the thickness of the boundary layer increases along the opposite running direction of the train, the pantograph fixing position would have significant influences on the aerodynamic characteristics of the pantographs and the train body [13]. In the present paper, aerodynamic models of high-speed trains with pantographs fixed on different positions are established based on the theory of computational fluid dynamics (CFD). The external flow fields of the high-speed trains are numerically simulated using the software STAR-CCM+. In addition, influences of the pantograph fixing position on the

(a)

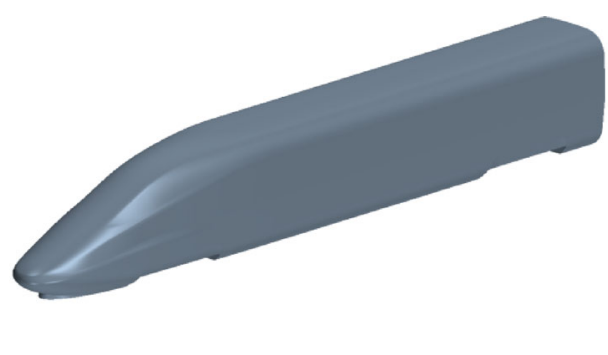

(b)

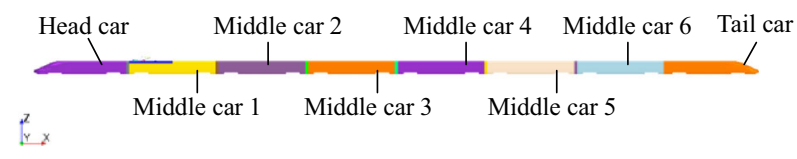

Fig. 1 Model of the head car (a) and the whole train (b)

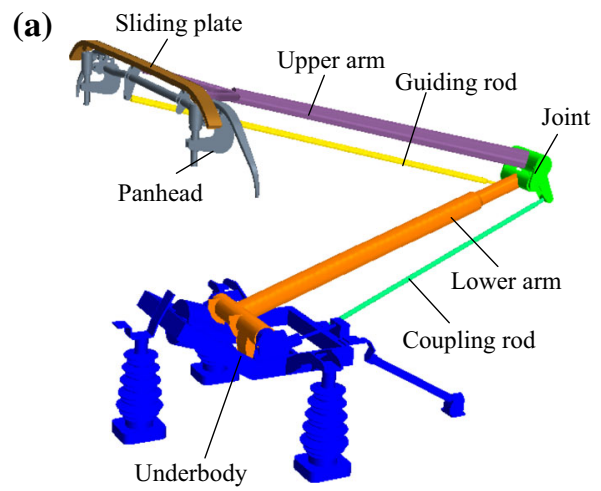

Fig. 2 Model of the pantograph (a) and the pantograph region (b) aerodynamic characteristics of the high-speed train and pantographs are analyzed.

\section{Computational model}

\subsection{Geometric model}

Based on a new-type high-speed train, a train model with eight cars (including a head car, six middle cars and a tail car) is established. The total length of the train is $200 \mathrm{~m}$. Figure 1 shows a simplified model of the head car and the whole train. The CX-PG pantograph is used for this study, which is a widely used pantograph in China Railway HighSpeed 380. The main components of the pantograph are reserved, and the cables and bolts are ignored. There are two pantographs in each position: One is lifted, and the other is folded. The model of the pantograph and the pantograph region is shown in Fig. 2.

\subsection{Computational domain and grids}

Figure 3 illustrates the computational domain of the flow field. The inlet of the computational domain extends $200 \mathrm{~m}$ ahead of the head nose, and the outlet is at a distance of $400 \mathrm{~m}$ from the tail nose. The height and width of the computational domain are 40 and $80 \mathrm{~m}$, respectively. The clearance between the bottom of train and the ground is $0.376 \mathrm{~m}$. The computational grids are built using the software STAR-CCM + , which consist of trimmed hexahedral elements, with 6 prismatic cell layers around the train (growth rate of 1.2). The thickness of the prismatic cell layer adjacent to the train wall is $0.5 \mathrm{~mm}$. Three refinement zones are defined around the train body and the pantographs. The minimum and maximum of the surface mesh size of pantographs are 2 and $20 \mathrm{~mm}$, respectively. The minimum and maximum of the surface mesh size of the train body are 20 and $80 \mathrm{~mm}$, respectively. The maximum volume mesh size

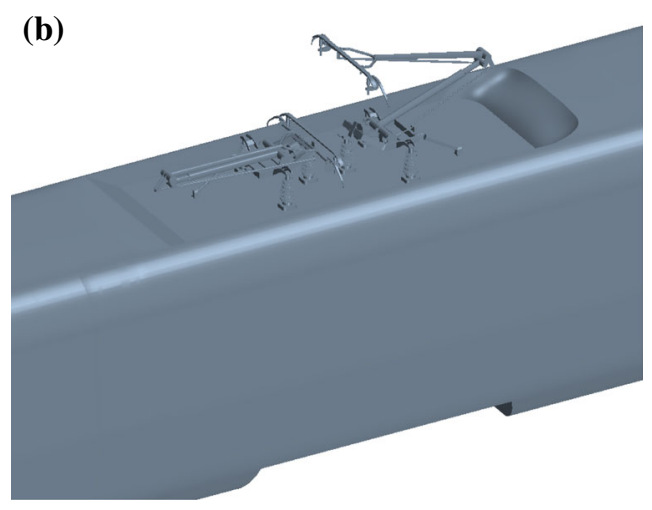


of the computational domain is $2000 \mathrm{~mm}$. The volume mesh sizes of the refinement zones around the train body and the pantographs are 60 and $20 \mathrm{~mm}$, respectively. Same mesh layouts are used for the train models with pantographs fixed on different positions. The amounts of computational grids in various cases are about 32.57-33.06 million. Partial grids of the train model are presented in Fig. 4.

\section{Numerical method and boundary conditions}

In this work, the train running speed is $97.22 \mathrm{~m} / \mathrm{s}$ $(350 \mathrm{~km} / \mathrm{h})$, and the Mach number is 0.286 , which is lower than 0.3. Therefore, the air compressibility can be ignored. The external flow fields around high-speed trains

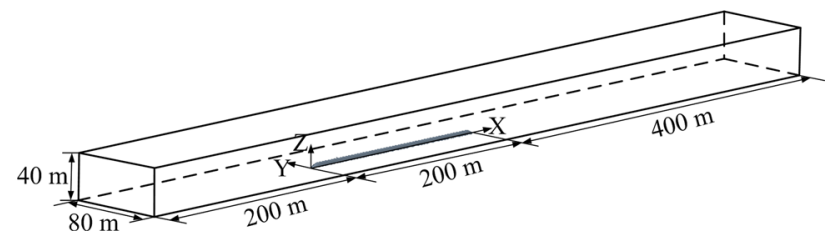

Fig. 3 Computational domain

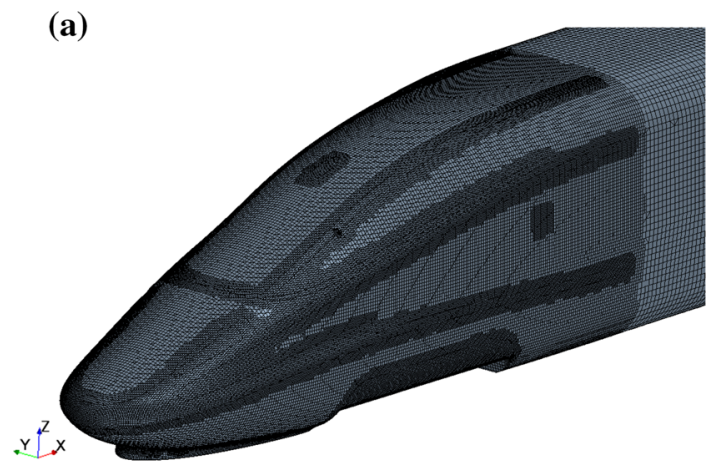

are simulated using 3D steady incompressible RANS equations. The Roe's FDS scheme and the lower-upper symmetric Gauss-Seidel (LU-SGS) method are selected for convective flux and temporal discretization, respectively. The $k-\omega$ SST (shear stress transport) model is adopted as the turbulent model. The standard wall functions are used near the wall to ensure the accuracy of the CFD results with a limited amount of mesh. The governing equation of the incompressible flow can be expressed as follows [14]:

$\operatorname{div}(\rho \boldsymbol{u} \varphi)=\operatorname{div}(\Gamma \operatorname{grad} \boldsymbol{\varphi})+\boldsymbol{S}$,

where $\rho$ is the air density, $\boldsymbol{u}$ is the velocity vector, $\boldsymbol{\varphi}$ is the flow flux, $\Gamma$ is the diffusion coefficient, and $S$ is the source item.

The boundary conditions of the computational domain are described below. The inlet is set as a velocity inlet boundary and the velocity magnitude is equal to the train running speed. The outlet is set as a pressure-outlet boundary and the gauge pressure on the outlet is $0 \mathrm{~Pa}$. The top and two sides of the domain are set as symmetry boundaries. The train body and pantographs are non-slip wall boundaries. In order to simulate the ground effect, the

(b)

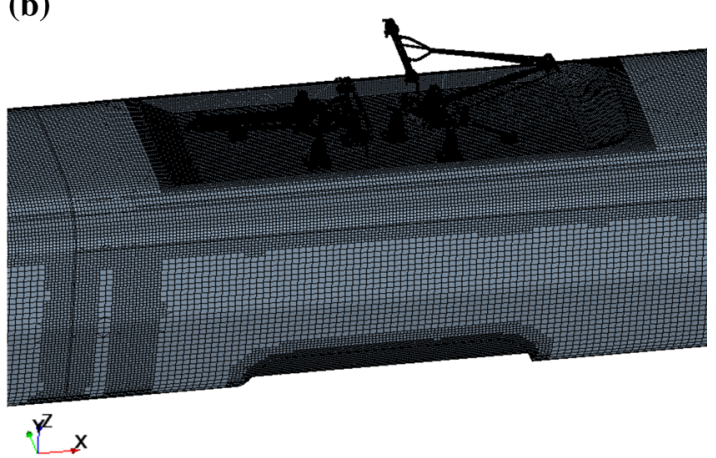

(c)

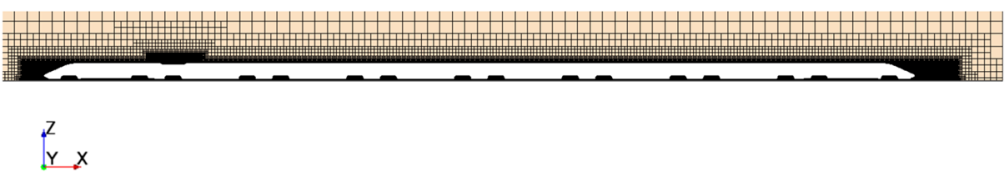

(d)
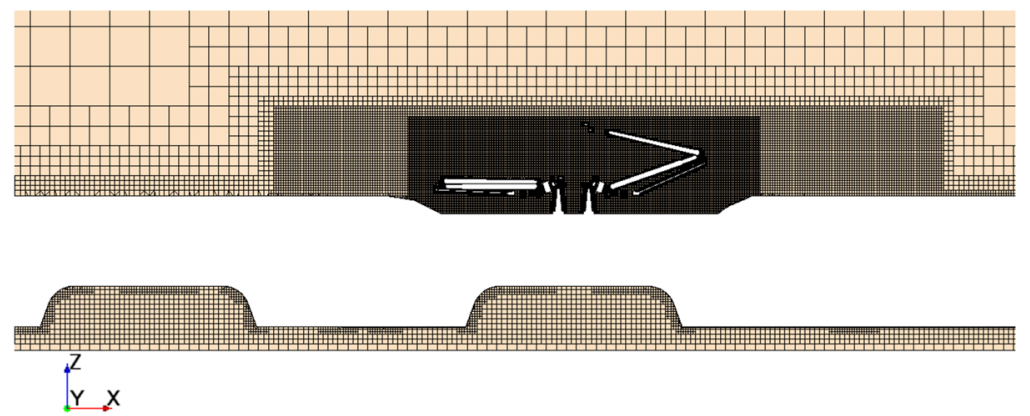

Fig. 4 Presentation of partial grids. a Surface mesh of streamlined head. b Surface mesh around pantograph region. c Longitudinal symmetry section mesh around train. d Closer view of symmetry section mesh around pantographs 


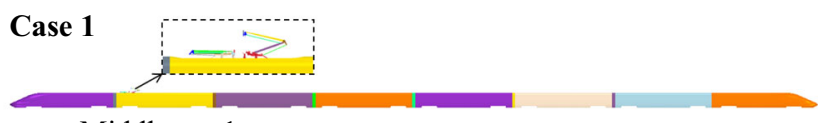

Middle car 1

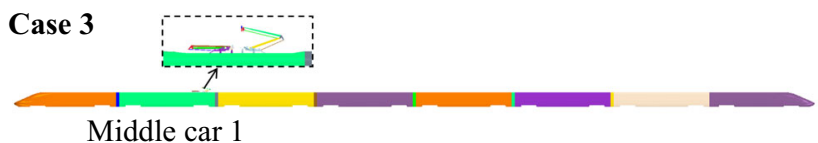

Middle car 1

Case 5

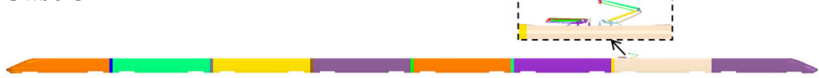

Middle car 6

\section{Case 7}

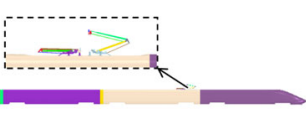

Middle car 6
Case 2

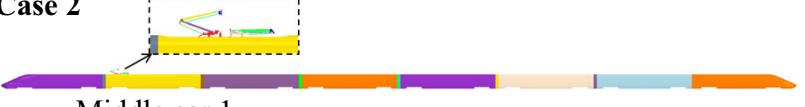

Middle car 1

Case 4

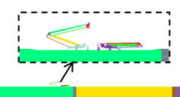

Middle car 1

Case 6

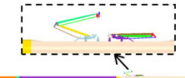

Middle car 6

Case 8

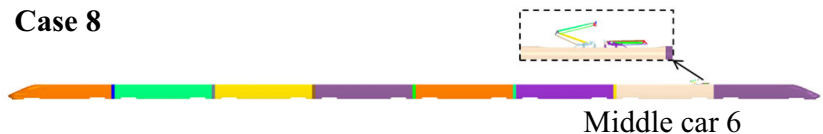

Fig. 5 Schematic diagram of the pantograph configurations
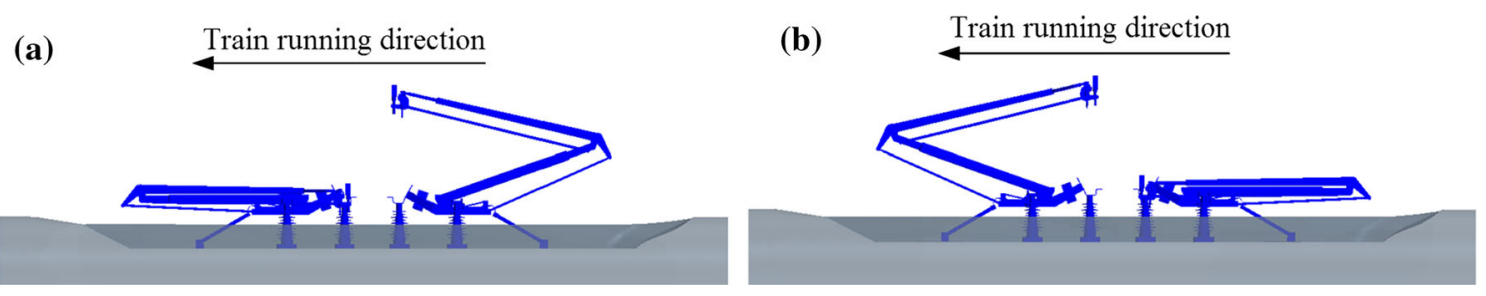

Fig. 6 Operational orientations of the pantographs. a Knuckle-downstream orientation. b Knuckle-upstream orientation

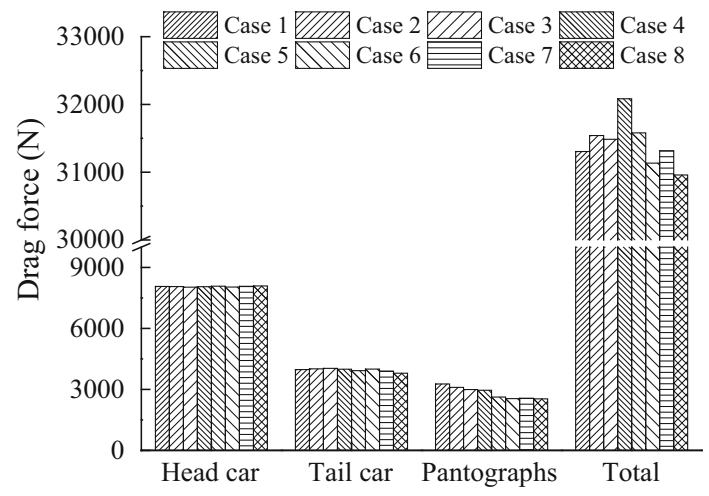

Fig. 7 Comparison of the drag forces in various cases

ground is set as a slip wall moving with the same speed as the inlet flow.

Four pantograph fixing positions are studied in this work: the front or the rear end of the first middle car and the front or the rear end of the last middle car. The schematic diagram of the pantograph configuration in each case is presented in Fig. 5. Two operational orientations of the lifted pantograph are considered in each position: the knuckle-downstream orientation and the knuckle-upstream orientation, as shown in Fig. 6.

\section{Results and discussion}

Figure 7 shows the comparison of the aerodynamic drag forces of high-speed trains in various cases, where the drag force of pantographs is the sum of the drag forces of the lifted pantograph and folded pantograph. It can be seen that the pantograph fixing position has little effect on the drag force of the head car, but has a large effect on the drag force of the tail car. The aerodynamic drag forces of the pantographs fixed on the last middle car are evidently smaller than those fixed on the first middle car. The main reason is that the thickness of the boundary layer around the last middle car is much larger than that around the first middle car, and the pantographs fixed on the last middle car are almost submerged in the boundary layer. Among the eight cases, the total aerodynamic drag force of the high-speed train in case 8 is the smallest and in case 4 is the largest.

Figure 8 shows the velocity contour around the pantographs in case 4 and case 8 . It can be seen that the velocity of the air flow around the pantographs in case 4 is larger than that in case 8 . As a result, the aerodynamic drag force of the pantographs in case 4 is larger than that in case 8.

The comparison of the aerodynamic lift forces of highspeed trains in various cases is shown in Fig. 9. It can be 

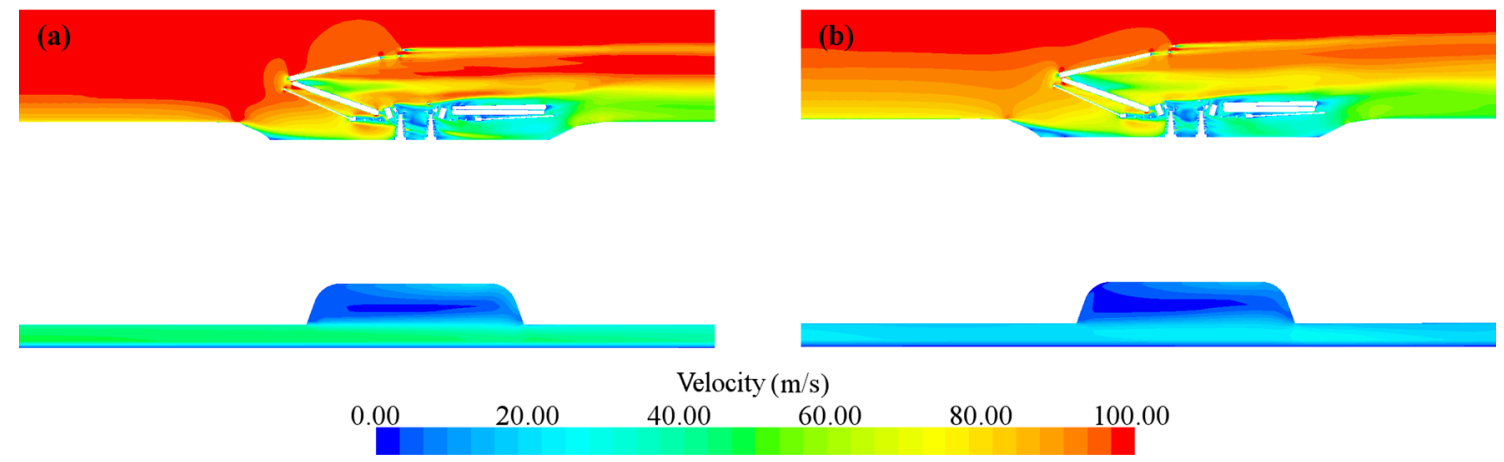

Fig. 8 Velocity contour around pantographs in case 4 (a) and case 8 (b)
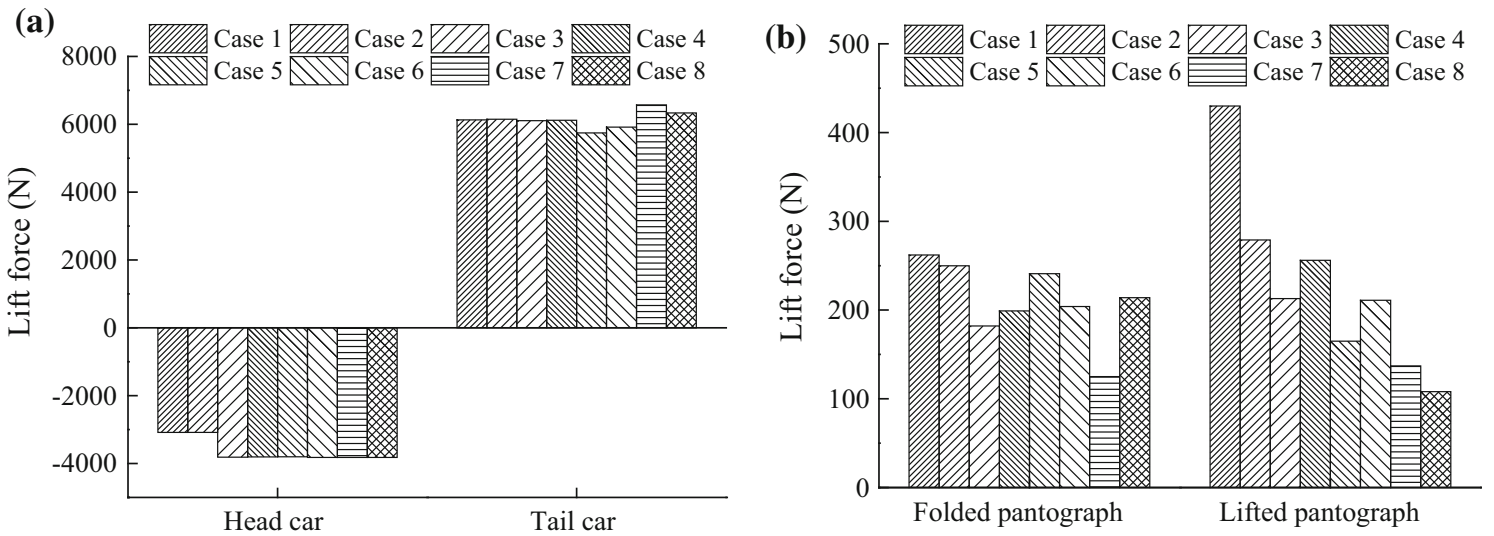

Fig. 9 Comparison of the lift forces in various cases. a Head car and tail car. b Pantographs

seen that the pantograph fixing position has a large effect on the aerodynamic lift forces of the head car and tail car. The absolute values of the aerodynamic lift forces of the head car in case 1 and case 2 are evidently smaller than those in other cases, while the aerodynamic lift forces of the tail car in case 7 and case 8 are evidently larger than those in other cases. The effects of the fixing position of pantographs on the aerodynamic lift forces of pantographs are obvious. The aerodynamic lift forces of the lifted pantographs fixed on the last middle car are smaller than those fixed on the first middle car, and the aerodynamic lift force of the lifted pantograph in case 8 is the minimum.

The aerodynamic lift force of the lifted pantograph directly influences the contact force between pantograph and catenary, and the contact state of the pantographcatenary system has a significant effect on the powercollecting capability of the pantograph. A too large contact force would lead to an abrasion increase in the pantograph and catenary. In contrast, a too small contact force would lead to an increase in the contact resistance between pantograph and catenary, resulting in heat generation, pantograph off-line, arc discharge, etc. As the sliding plate of the lifted pantograph contacts with the catenary directly, it is more reasonable to analyze the lift force of the sliding plate when considering the interaction between pantograph and catenary. Figure 10 shows the aerodynamic lift forces acting on the sliding plates of the

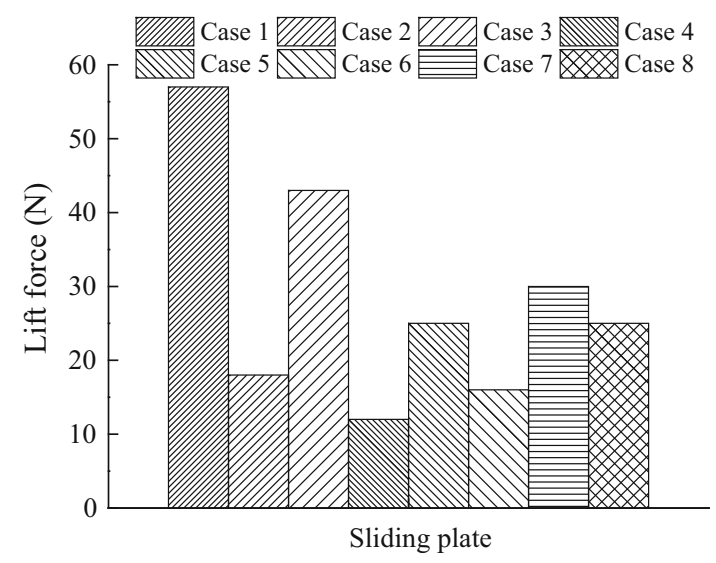

Fig. 10 Aerodynamic lift forces of the sliding plates of the lifted pantographs in various cases 
lifted pantographs in various cases. It can be seen that the pantograph fixing position has a considerable influence on the lift force of sliding plates. The lift force of the sliding plate of the lifted pantograph in case 1 is the maximum and in case 4 is the minimum. However, the total aerodynamic drag force of the train in case 4 is the maximum (Fig. 7). The lift forces of the sliding plates of the lifted pantographs fixed on the last middle car (i.e., cases 5-8) are relatively small, and their differences between each other are within $15 \mathrm{~N}$.

From the above analysis, it can be concluded that considering the total aerodynamic drag force of the train and the aerodynamic lift force of the lifted pantograph, when the pantographs are fixed on the rear end of the last middle car and the lifted pantograph is in the knuckle-upstream orientation, the aerodynamic performance of the highspeed train is the best.

\section{Conclusions}

In this paper, the aerodynamic performances of high-speed trains with pantographs fixed on different positions are calculated based on the theory of CFD. The following conclusions can be drawn:

(1) The pantograph fixing position has little effect on the aerodynamic drag force of the head car and has a large effect on the aerodynamic drag force of the tail car.

(2) When pantographs are fixed on the rear end of the last middle car and the lifted pantograph is in the knuckleupstream orientation, the total aerodynamic drag force of the high-speed train is the minimum.

(3) The pantograph fixing position has a significant influence on the aerodynamic lift forces of the head car and the tail car. The absolute values of the aerodynamic lift forces of the head car of a highspeed train with the pantographs fixed on the front end of the first middle car are evidently smaller than those in other cases.

(4) The influences of the fixing position on the aerodynamic lift forces of pantographs are obvious. The aerodynamic lift forces of the lifted pantographs fixed on the last middle car are smaller than those fixed on the first middle car.

(5) Considering the total aerodynamic drag force of the train and the aerodynamic lift force of the lifted pantograph, when the pantographs are fixed on the rear end of the last middle car and the lifted pantograph is in the knuckle-upstream orientation, the aerodynamic performance of the high-speed train is the best.
Acknowledgements This work was supported by the High-Speed Railway Basic Research Fund Key Project of China (Grant No. U1234208) and the National Natural Science Foundation of China (Grant Nos. 51475394 and 51605397).

Open Access This article is distributed under the terms of the Creative Commons Attribution 4.0 International License (http:// creativecommons.org/licenses/by/4.0/), which permits unrestricted use, distribution, and reproduction in any medium, provided you give appropriate credit to the original author(s) and the source, provide a link to the Creative Commons license, and indicate if changes were made.

\section{References}

1. Raghunathan RS, Kim HD, Setoguchi T (2002) Aerodynamics of high-speed railway train. Prog Aerosp Sci 38(6):469-514

2. Zheng XH, Zhang JY, Zhang WH (2011) Numerical simulation of aerodynamic drag for high-speed train bogie. J Traffic Transp Eng 11(2):45-51 (in Chinese)

3. Liu X, Deng J, Zheng Y et al (2013) Impact of aerodynamics of pantograph of a high-speed train on pantograph-catenary current collection. J Zhejiang Univ-Eng Sci 47(3):558-564 (in Chinese)

4. Chater E, Ghani D, Giri F et al (2015) Output feedback control of pantograph-catenary system with adaptive estimation of catenary parameters. J Mod Transp 23(4):252-261

5. Wu G, Wei W, Gao G et al (2016) Evolution of the electrical contact of dynamic pantograph-catenary system. J Mod Transp 24(2):132-138

6. Zhang JH, Yang J, Yao Y (2010) Wind tunnel test investigation on pantograph drag reduction of high speed train. J Railw Sci Eng 7(6): 116-121 (in Chinese)

7. Guo DL, Yao SB, Liu CH et al (2012) Unsteady aerodynamic characteristics of high-speed pantograph. J China Railw Soc 34(11):16-21 (in Chinese)

8. Li RP, Zhou N, Zhang WH et al (2012) Calculation and analysis of pantograph aerodynamic uplift force. J China Railw Soc 34(8):26-32 (in Chinese)

9. Fu SQ, Chen DW, Liang JY et al (2013) Investigation on wind tunnel tests of aerodynamic characteristics of high-speed pantograph. Railw Locomot Car 33(3):123-126 (in Chinese)

10. Pombo J, Ambrosio J, Pereira M et al (2009) Influence of the aerodynamic forces on the pantograph-catenary system for highspeed trains. Veh Syst Dyn 47(11):1327-1347

11. Lee Y, Rho J, Kim KH et al (2015) Experimental studies on the aerodynamic characteristics of a pantograph suitable for a highspeed train. Proc Inst Mech Eng Part F J Rail Rapid Transit 229(2):136-149

12. Du J, Liang JY, Tian AQ (2015) Analysis of aerodynamic characteristics for pantograph of high-speed trains. J Southwest Jiaotong Univ 50(5):935-941 (in Chinese)

13. Ito $M$ (2000) Improvement to the aerodynamic characteristics of Shinkansen rolling stock. Proc Inst Mech Eng Part F J Rail Rapid Transit 214(3): 135-143

14. Li T, Zhang JY, Zhang WH (2011) An improved algorithm for fluid-structure interaction of high-speed trains under crosswind. J Mod Transp 9(2):75-81 